\title{
The Relationship between Musical Pitch and Temporal Responses of the Auditory Nerve Fibers
}

\author{
Kengo Ohgushi ${ }^{1)}$ and Yukiko Ano ${ }^{2)}$ \\ 1) Emeritus Professor, Kyoto City University of Arts \\ 2) Flutist
}

\begin{abstract}
To investigate how the high pitched notes in a musical score are played on the piccolo, nine flutists produced tones of a $\mathrm{C}$ major scale, from $\mathrm{C} 6$ to $\mathrm{C} 8$, using their own piccolo. The fundamental frequency of each tone was measured. The results showed that all tones were produced higher in frequency than the theoretical values and that this tendency was striking in the higher frequency range. This phenomenon is discussed in terms of temporal responses of auditory nerve fibers. J Physiol Anthropol Appl Human Sci 24(1): 99-101, 2005 http://www.jstage.jst.go.jp/browse/jpa
\end{abstract}

Keywords: pitch, musical pitch, piccolo, auditory nerve fiber, interspike interval

\section{Introduction}

In music theory, the frequency ratio relationships between any two notes in a musical scale within an octave are maintained in the other octaves. However, it is known that in the average piano tuning, tones above $\mathrm{C} 6$ are tuned higher than the equally tempered scale (Martin and Ward, 1961). The amount of the deviation is $1-2$ cents for C6, 10 cents for $\mathrm{C} 7$, and 30 cents for $\mathrm{C} 8$. This phenomenon is at least partially accounted for by the inharmonic partials of piano tones (Rossing, 1990). However, it has also been reported that the subjective octave is slightly larger than the physical octave for pure tones (Ward, 1954; Ohgushi, 1983; Hartmann, 1993). This octave enlargement phenomenon is especially pronounced in the high-frequency range. This suggests that pure tones in the high-frequency range have also pitches that slightly deviate from the equally tempered scale. It seems possible that this deviation is common to piano tones with their inharmonic partials, to pure tones, and to musical instrument tones with harmonic partials.

On the other hand, piccolo players (in general flutists play the piccolo in the orchestra) often have difficulty in playing tones with a correct pitch in the high-frequency range. The piccolo is about one-half the length of the flute, and it sounds an octave higher than the flute. Experienced piccolo players know that their performed pitches are often higher than the theoretical values for the fundamental, as measured by an electronic tuner. We measured the fundamental frequencies of performed notes to confirm the observations of expert flutists and to obtain quantitative data.

\section{Method}

Nine flutists performed tones of the $\mathrm{C}$ major scale, from $\mathrm{C} 6$ to $\mathrm{C} 8$, on their own piccolos. A standard A4 tone $(442 \mathrm{~Hz})$ was presented to the players by a piano before playing. The flutists played long successive tones of 2-3 s without vibrato in a silent room. The frequency measurement was carried out by measuring the period of the waveform. Stable portions, where frequency fluctuations were small, were used for the measurement.

\section{Results}

Figure 1 shows the average data over nine players. The data represents the frequency of the performed tone as a function of pitch name. The abscissa represents the pitch name from C6 to C8. The ordinate shows the average frequency deviation, expressed in cents, of each measured frequency from the theoretical value, where the theoretical value is calculated based on the equal tempered scale with A4 of $442 \mathrm{~Hz}$.

This result shows that all tones were performed higher in frequency than the theoretical value and that this tendency is striking in the higher frequency range. As a result, the empirical observation that the indication on an electronic tuner does not fit the perceived pitch in the high-frequency range was confirmed.

\section{Discussion}

Despite the music theory in which the frequency ratios between any two notes on a musical scale within an octave should be maintained in the other octaves, the frequency 


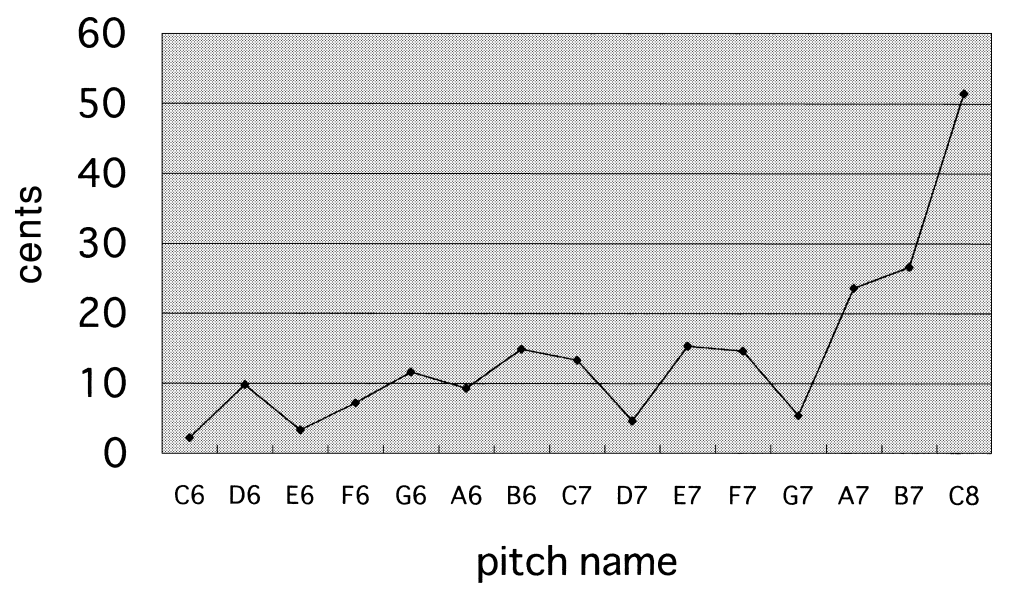

Fig. 1 The fundamental frequency deviation of the performed tone as a function of pitch name. Nine flutists produced tones consisting of $\mathrm{C}$ major scale tones, from $\mathrm{C} 6$ to $\mathrm{C} 8$, on their own piccolo. The ordinate shows the frequency deviation in cents, of each frequency from the theoretical value.

measurements of performed notes on the piccolo showed a rather large upward deviation from the theoretical values, on the average, in the high-frequency range. This result suggests that the relation between frequency and perceived musical pitch does not hold in the frequency range above C6. We will discuss a possible neurophysiological explanation of the frequency deviation from the theoretical value.

Musical pitch is mainly based on the period of an acoustic stimulus. An acoustic stimulus is transformed in the inner ear into neural impulses which are conducted by the auditory nerve fibers. The auditory nerve fibers discharge impulses corresponding to particular phases of the stimulating waveform for low frequency tones. The temporal distribution of these impulses can be represented in the form of an interspike interval (ISI) histogram. Figure 2 shows an example of such a histogram for impulses from a single auditory nerve fiber of a squirrel monkey when a pure tone of $1,000 \mathrm{~Hz}$ was presented (Rose et al., 1967). The abscissa represents the interspike interval with a binwidth of $0.1 \mathrm{~ms}$, and the ordinate indicates the number of experimentally measured intervals in each bin. This interval histogram is polymodal, and the first modal value corresponds approximately to the period of the tone. It was observed that the period of the tone divided by the first modal value was not exactly 1.0 , but depends on the frequency of the tone. These ratios were calculated (Ohgushi, 1978) from the data for four auditory nerve fibers published by Rose et al. (1967, 1968). The calculated result clearly shows that these values decrease with increasing frequency of the tone (Ohgushi, 1978, 1983).

The interspike interval corresponding to successive cycles of the stimulating waveform is an important cue for pitch perception. The octave enlargement phenomenon was explained by the fact that the period of the tone divided by the first modal value becomes smaller in the high-frequency range (Ohgushi, 1983). The fact that the value decreases with increasing frequency also suggests that the musical pitch shifts upward in the high-frequency range.

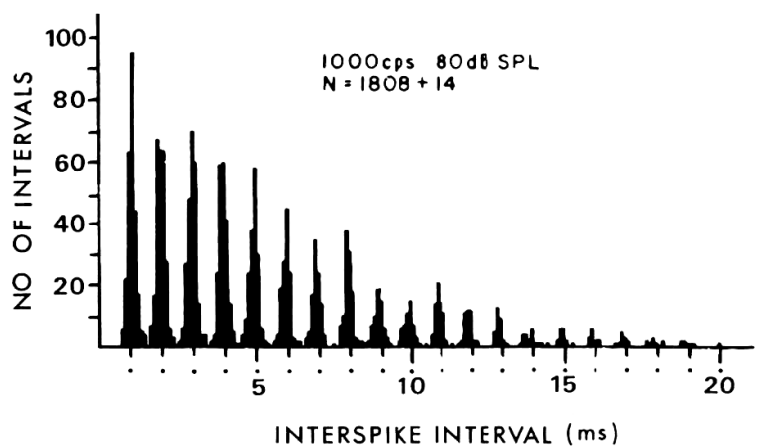

Fig. 2 An example of an interspike interval histogram of impulses from a single auditory nerve fibers of a squirrel monkey when a pure tone of $1,000 \mathrm{~Hz}$ was presented (Rose et al., 1967).

As stated above, in well-tuned pianos, tones above $\mathrm{C} 6$ are tuned slightly sharp compared to equal temperament. The inharmonicity in piano strings has been considered to be the main reason for this "stretch-tuned" (Rossing, 1990). However, the tendency in pianos is almost the same as the data in Fig. 1, where there is no problem of inharmonicity. This suggests that deviation from equal temperament in the high-frequency range is primarily due to the process of transmission in the auditory nervous system rather than the inharmonicity in piano strings.

Acknowledgements This work was supported by Grant No. 14101001 from the JSPS.

\section{References}

Hartmann WM (1993) On the origin of the enlarged melodic octave. J Acoust Soc Am 93: 3400-3409

Martin DW, Ward WD (1961) Subjective evaluation of musical scale temperament in pianos. J Acoust Soc Am 33: 582-585

Ohgushi K (1978) On the role of spatial and temporal cues in the perception of the pitch of complex tones. J Acoust Soc 
Am 64: 764-771

Ohgushi K (1983) The origin of tonality and a possible explanation of the octave enlargement phenomenon. J Acoust Soc Am 73: 1694-1700

Rose JE, Brugge JF, Anderson DJ, Hind JE (1967) Phaselocked response to low-frequency tones in single auditory nerve fibers of the squirrel monkey. J Neurophysiol 30 : 769-793

Rose JE, Brugge JF, Anderson DJ, Hind JE (1968) Patterns of activity in single auditory nerve fibers of the squirrel monkey. In de Euck AVS, Knight $\mathrm{J}$ eds. Hearing Mechanisms in Vertebrates. Churchill, London, 144-168
Rossing TR (1990) The science of sound. Addison-Wesley Publishing Company, 290-292

Ward WD (1954) Subjective musical pitch. J Acoust Soc Am 26: $369-380$

Received: September 22, 2004

Accepted: October 22, 2004

Correspondence to: Kengo Ohgushi, 1-6-10 Oheyama, Goryo, Nishikyo-ku, Kyoto 610-1102, Japan

Phone: +81-75-331-1524

e-mail: ohgushi@kcua.ac.jp 